\title{
Improving anesthesia care and pain medicine in rural Canada: nothing about us without us
}

\author{
Beverley A. Orser, MD, PhD, FRCPC, FCAHS, FRSC (D) C. Ruth Wilson, MD, CCFP, FCFP • \\ Daniel Bainbridge, MD, FRCPC
}

Received: 16 July 2021/Revised: 16 July 2021/ Accepted: 16 July 2021/Published online: 20 September 2021

(C) Canadian Anesthesiologists' Society 2021

Attention has recently focused on inequitable access to and poorer outcomes following surgical and obstetric care for rural and Indigenous people in Canada. ${ }^{1,2}$ Anesthesia is a foundation for the provision of these services. Small rural hospitals continue to experience closures of their surgical and obstetric services, partly due to a shortage of healthcare providers-or perhaps we should say a misdistribution of healthcare providers. Approximately $18 \%$ of Canadians live in rural areas, but only $8 \%$ of Canada's physicians work in these regions. ${ }^{3,4}$ Anesthesia services are not immune to this problem. ${ }^{4}$ As just one example, waits of up to a year for anesthetic services in northern Canada have contributed to shortages in essential dental procedures for children and as of summer 2021, up to 1,000 children were waiting for dental care in Nunavut. ${ }^{5}$

A symposium organized by the Department of Anesthesiology and Pain Medicine at the University of Toronto provided an opportunity to discuss issues, strategies, and interventions pertaining to the delivery of anesthesia care and pain medicine in rural and remote regions of Canada. ${ }^{6}$ Over two days, anesthesiologists,

B. A. Orser, MD, PhD, FRCPC, FCAHS, FRSC ( $\square)$

Department of Anesthesiology and Pain Medicine, Temerty

Faculty of Medicine, University of Toronto, Room 3318,

Medical Sciences Building, 1 King's College Circle, Toronto,

ON M5S 1A8, Canada

e-mail: beverley.orser@utoronto.ca

C. R. Wilson, MD, CCFP, FCFP

Department of Family Medicine, Queen's University, Kingston, ON, Canada

D. Bainbridge, MD, FRCPC

Department of Anesthesia and Perioperative Medicine, Schulich School of Medicine and Dentistry, Western University, London, ON, Canada family physicians, surgeons, obstetricians, nurses, midwives, and medical trainees from coast to coast to coast gathered virtually to participate. Collectively, they acknowledged the need to improve the provision of care in non-urban centres and expressed a commitment to finding solutions. A "call to action", in the form of a consensusbased report, was generated. ${ }^{7}$ The topics of the workshop and authors of the symposium report are presented below, and several of the lectures are available for viewing. ${ }^{6}$

One major strength of this symposium lay in the broad representation from different healthcare professions and the contributions of those who are themselves living in rural and remote communities of Canada, including Yellowknife, Iqaluit, and Fort Simpson. Conversely, a limitation of the symposium was the lack of representation from patients and community groups. This limitation is important because a central theme that emerged was that any recommendations must focus on the patient and community, rather than on medical specialties, academic programs, governments, or professional groups. Indeed, at the opening of the event, Dean Trevor Young of the Temerty Faculty of Medicine, University of Toronto stated, "We in Toronto cannot presume to know-though with the best of intentions and insights-what is best for [rural and remote communities]." 6 These comments align with the adage "Nothing about us without us."

Dean Young's statement and this slogan remind us that we should not develop policies without meaningful participation of the groups who will be affected by those policies. In Canada, increasing evidence abounds of the tragic outcomes of ill-informed and misguided policies that ignored the importance of self-determination and empowerment of patient groups, communities, and the sovereignty of Indigenous peoples. The design and development of interventions intended to serve the needs 
of rural communities require substantial input from those communities and from local healthcare providers.

Discussions during the symposium focused on five central themes, one of which was the need to obtain comprehensive data to inform evidence-based responses. Such data can overcome information deficits or perceptions informed by anecdotes rather than evidence. It was also apparent that no single organization or group has the insights, expertise, or capacity required to identify and implement potential solutions. Collaboration with a broad "coalition of the willing" is required to ensure the success of national strategies. In addition, mentorship and peer support are required to foster the well-being and professional development of healthcare providers and to acknowledge the value of their work.

The workshops, lectures, and discussions resulted in the following recommendations, as set out in the symposium report:

1. Academic health science centres must accept their social accountability mandate to educate a sufficient quantity and quality of both specialist and nonspecialist anesthesia providers to meet Canada's needs.

2. Health human resource planning for anesthesiology and pain medicine must become more coordinated, data-driven, and based on population-needs assessments.

3. Mentoring and coaching of anesthesia providers in rural and remote communities must be prioritized as an immediate practical step to support physician recruitment and retention.

4. Institutions and organizations must take advantage of the possibilities that technology offers for remote coaching, including virtual and augmented virtual reality, as well as the building of a remote clinical service presence.

5. Regional networks of care must support the crucial role of small, active rural hospitals. Anesthesia, surgery, and maternity care in these rural programs are tightly integrated and mutually interdependent and must therefore receive parallel support.

6. Current initiatives of professional organizations should involve wider partnerships to move ahead with implementing these recommendations.

Of course, no single solution can fulfill the needs of all rural communities. In Canada, healthcare is managed at the provincial and territorial level. Unfortunately, many provinces and territories lack the infrastructure and resources to fully support rural healthcare providers. Thus, it is imperative that national organizations become involved to help support physicians. In addition, individual regions within the country will need to identify their own solutions to increase access to care in rural and remote communities. For example, the province of Quebec currently provides anesthesia services almost exclusively through coverage by specialty-trained anesthesiologists, whereas some other provinces are hoping to increase provision of services by expanding both their specialty and family practice anesthesia programs. In addition, certified clinical anesthesia assistants also play an important role in expanding high-level clinical services.

Provincial and national medical organizations, such as the College of Family Physicians of Canada (CFPC), the Royal College of Physicians and Surgeons of Canada, and the Canadian Anesthesiologists' Society (CAS), need to engage in addressing the shortages of anesthesia healthcare providers in rural and remote regions. These organizations are responsible for providing or accrediting training and continuing education programs. They are also involved in setting guidelines for care, which help to ensure the safety of anesthetic services. Certainly, the maintenance of clinical competencies can be challenging, especially in remote locations. There are also challenges in understanding needs of rural anesthesia providers to ensure the relevance of educational materials provided. Investment in new technologies, such as online platforms, would help to address some of these issues. Coordination of the various stakeholder organizations to prevent duplication of support or, worse, failure of support because of poor communication among providers is crucial. Creating regular opportunities for discussion among representatives of provincial and national bodies and local healthcare providers will be key.

A team will be established to create a work plan based on the symposium's recommendations and then put the plan into action. One organization that could provide a "home" for such a program is the Collaborative Advisory Group for General and Family Practice Anesthesia (CAGA). Formed more than 20 years ago in response to the need for increased anesthesia services in rural Canada, CAGA's goal is to support family physicians by giving them additional skills in anesthesia. It is a multidisciplinary group comprising representatives from the CAS, the CFPC, the Society of Rural Physicians of Canada (SRPC), the Association of Canadian University Departments of Anesthesia, and the directors of family practice anesthesia residency programs. Jointly funded by the CAS, the CFPC, and the SRPC, CAGA clearly recognizes the need for a renewed commitment to and investment in rural services.

Efforts have continued since the symposium. For example, anesthesiologists at St. Paul's Hospital in Vancouver, British Columbia, are partnering with family practice anesthetists in northern British Columbia to provide real-time training in the provision of ultrasoundguided adductor canal nerve blocks by means of remote 
videoconferencing coupled with real-time supervision. ${ }^{8}$ This program builds on a robust model that has been supporting the ongoing training of family practice anesthesia providers in British Columbia. ${ }^{9}$ As a second example, Dr. Ivy Bourgeault and Dr. Sarah Simkin and the Canadian Health Workforce Network are undertaking a quantitative review of the anesthesia workforce for the period of 1996 to 2018, using data from the Canadian Institute for Health Information. ${ }^{10,11}$ Also, investigators at Sunnybrook Health Sciences Centre in Ontario have created a platform to conduct live coaching of rural and remote anesthesiologists using a virtual-reality- coupled with an augmented-reality telesimulation software called HoloSIM. ${ }^{12}$ These technologies allow distant users to learn from facilitators in a different geographic location. Operating rooms can instantly be converted into a highfidelity simulation centre by projecting interactive holographic images of patients. Scenarios can be controlled and monitored from a distance by the coach, thereby reducing the equipment, staff, and space barriers to simulation.

Overall, the mandate is clear. Nevertheless, substantive changes to the provision of anesthesia and pain medicine in rural and remote regions of Canada will not be possible without adequate provincial funding. Such investments must include increasing the number of training positions for both family practice anesthesia providers and specialist anesthesiologists. Furthermore, the various stakeholdersmost notably governments-must not unilaterally develop solutions to the shortage of anesthesia care providers without appropriate and meaningful consultation with patients, local healthcare providers, and physician groups. Finally, academic departments must train physicians who are committed to working in rural communities. Considerable challenges lie ahead. Nevertheless, as the Right Honourable Julie Payette, then Governor General of Canada, commented in her opening remarks for the symposium, "We can do better, and we are actually better equipped to solve this problem than ever before, so we must take every opportunity to tackle this persistent problem." 6

\section{Anesthesia workforce planning in urban and rural Canada:}

Ivy Bourgeault, Mateen Raazi, Sarah Simkin, Pip Swartz
Coaching networks, mentorship, and educational opportunities:

Kirk McCarroll, Gary Morris, Elaine Ng, Margaret Tromp, Hira Raheel

Policy support for rural anesthesia and pain medicine:

Lee Errett, Dietrich Furstenburg, Vaibhav Kamble, John McAlpine, Taryn Davidson

Technology, virtual reality, and distance coaching and learning:

Fahad Alam, Sarah Newbery, Ahtsham Niazi, Julian Wiegelmann, Julian DeBacker

Training and supporting rural generalists (anesthesia, obstetrics, surgery):

Ryan Falk, Stu Iglesias, Aviva Stewart, Enrich Van der Linde, Miguel Proulx

Rural and remote practice models of care in Canada, including allied health professionals:

Roy Kirkpatrick, Matthew Kurrek, Jason McVicar, Roanne Preston, Alborz Noorani

Policy through collaboration with national medical societies and government:

Angela Enright, Colin McCartney, Robert Milkovich, Victor Ng, Tarimobo Otobo

\section{L'amélioration des soins d'anesthésie et de médecine de la douleur dans les régions rurales du Canada : rien sur nous sans nous}

L'accès inéquitable aux soins chirurgicaux et obstétricaux pour les populations rurales et autochtones du Canada, et les issues plus défavorables qui en découlent, ont récemment fait l'objet d'une attention particulière. ${ }^{1,2}$ L'anesthésie est l'un des fondements de la prestation de 
ces services. Les petits hôpitaux ruraux continuent de connaitre des fermetures de leurs services chirurgicaux et obstétricaux, en partie en raison d'une pénurie de travailleurs de la santé, ou peut-être devrions-nous dire d'une mauvaise répartition des travailleurs de la santé. Environ $18 \%$ des Canadiens vivent dans des régions rurales, mais seulement $8 \%$ des médecins canadiens travaillent dans ces régions. ${ }^{3,4}$ Les services d'anesthésie ne sont pas à l'abri de ce problème. ${ }^{4}$ À titre d'exemple, les délais d'attente pouvant aller jusqu'à un an pour des services d'anesthésie dans le Nord du Canada ont contribué à la pénurie d'interventions dentaires essentielles pour les enfants et, à l'été 2021, jusqu'à 1000 enfants étaient en attente de soins dentaires au Nunavut. ${ }^{5}$

Un symposium organisé par le Département d'anesthésiologie et de médecine de la douleur de l'Université de Toronto a été l'occasion de discuter des enjeux, des stratégies et des interventions liés à la prestation de soins d'anesthésie et de médecine de la douleur dans les régions rurales et éloignées du Canada. ${ }^{6}$ Pendant deux jours, les anesthésiologistes, médecins de famille, chirurgiens, obstétriciens, infirmières, sagesfemmes et résidents en médecine d'un océan à l'autre se sont réunis virtuellement. Collectivement, ils ont reconnu la nécessité d'améliorer la prestation des soins dans les centres non urbains et se sont engagés à trouver des solutions. Un «appel à l'action », prenant la forme d'un rapport consensuel, est né de ce symposium. ${ }^{7}$ Les thèmes de l'atelier et les auteurs du rapport du symposium sont présentés ci-dessous, et plusieurs des conférences sont disponibles pour visionnement. ${ }^{6}$

L'une des principales forces de ce symposium est liée à la vaste représentation de différentes professions de la santé et aux contributions de celles et ceux qui vivent dans des collectivités rurales et éloignées du Canada, y compris à Yellowknife, Iqaluit et Fort Simpson. En revanche, l'une des limites du symposium résidait dans le manque de représentation des patients et des groupes communautaires. Cette lacune est importante : en effet, l'un des thèmes centraux qui s'est dégagé était que toute recommandation devra se concentrer sur le patient et la communauté, plutôt que sur les spécialités médicales, les programmes universitaires, les gouvernements ou les groupes professionnels. En effet, lors de l'ouverture de l'événement, Trevor Young, le doyen de la Faculté de médecine Temerty de l'Université de Toronto, a déclaré : « À Toronto, nous ne pouvons pas présumer savoir, malgré nos meilleures intentions et perspectives, ce qui est le mieux pour [les collectivités rurales et éloignées]. » ${ }^{6} \mathrm{Ces}$ commentaires sont en adéquation avec l'adage « Rien sur nous sans nous ».

La déclaration de Dr Young et ce slogan nous rappellent que nous ne devrions pas élaborer de politiques sans la participation significative des groupes qui seront touchés par ces mêmes politiques. Au Canada, les données abondent chaque jour un peu plus quant aux conséquences tragiques de politiques mal informées et malavisées qui n'ont pas tenu compte de l'importance de l'autodétermination et de l'émancipation des groupes de patients, des communautés et de la souveraineté des peuples autochtones. La conception et l'élaboration d'interventions visant à répondre aux besoins des collectivités rurales exigent une importante contribution de ces collectivités et des travailleurs de la santé locaux.

Les discussions au cours du symposium ont porté sur cinq thèmes centraux, dont l'un était la nécessité d'obtenir des données exhaustives pour éclairer des réponses fondées sur des données probantes. De telles données peuvent permettre de combler les lacunes en matière d'information ou les perceptions fondées sur des anecdotes plutôt que sur des données probantes. Il était également évident qu'aucun organisme ou groupe ne possédait les connaissances, l'expertise ou la capacité nécessaires à identifier et mettre en œuvre, seul, des solutions potentielles. La collaboration d'une large " coalition de volontaires » est indispensable pour garantir le succès de stratégies nationales. De plus, le mentorat et le soutien des pairs sont nécessaires pour favoriser le bien-être et le perfectionnement professionnel des travailleurs de la santé ainsi que pour reconnaître la valeur de leur travail.

Des ateliers, conférences et discussions ont découlé les recommandations suivantes, telles qu'elles figurent dans le rapport du symposium :

7. Les centres universitaires de sciences de la santé doivent accepter leur mandat d'imputabilité sociale afin d'éduquer un nombre suffisant de prestataires d'anesthésie spécialisés et non spécialisés suffisamment bien pour répondre aux besoins du Canada.

8. La planification des ressources humaines en santé pour l'anesthésiologie et la médecine de la douleur doit devenir plus coordonnée, axée sur les données et fondée sur l'évaluation des besoins de la population.

9. Le mentorat et l'encadrement des prestataires de services d'anesthésie dans les collectivités rurales et éloignées doivent être priorisés en tant qu'étape pratique immédiate pour appuyer le recrutement et la rétention des médecins.

10. Les institutions et les organismes doivent tirer parti des possibilités qu'offre la technologie pour l'encadrement à distance, y compris la réalité virtuelle et augmentée, ainsi que la mise sur pied de services cliniques à distance.

11. Les réseaux régionaux de soins doivent soutenir le rôle crucial des petits hôpitaux ruraux en activité. 
Dans ces programmes ruraux, l'anesthésie, la chirurgie et les soins de maternité sont étroitement liés et interdépendants et doivent donc recevoir un soutien parallèle.

12. Les initiatives actuelles des organisations professionnelles devraient faire appel à des partenariats plus étendus pour aller de l'avant avec la mise en œuvre de ces recommandations.

Bien entendu, il n'existe pas de solution unique pour répondre aux besoins de toutes les communautés rurales. Au Canada, les soins de santé sont gérés à l'échelle provinciale et territoriale. Malheureusement, bon nombre de provinces et de territoires ne disposent ni de l'infrastructure, ni des ressources nécessaires pour soutenir pleinement les prestataires de soins de santé en milieu rural. Il est donc impératif que les organismes nationaux s'impliquent pour soutenir les médecins. De plus, chaque région devra trouver ses propres solutions pour accroître l'accès aux soins dans les collectivités rurales et éloignées. Par exemple, la province du Québec offre actuellement des services d'anesthésie presque exclusivement par l'entremise d'anesthésiologistes spécialisés, tandis que d'autres provinces espèrent accroître la prestation de services en élargissant tant leurs programmes d'anesthésie spécialisée que leurs programmes d'anesthésie en médecine familiale. En outre, les assistants en anesthésie clinique certifiés jouent eux aussi un rôle important dans l'expansion des services cliniques de haut niveau.

Les organisations médicales provinciales et nationales, telles que le Collège des médecins de famille du Canada (CMFC), le Collège royal des médecins et chirurgiens du Canada et la Société canadienne des anesthésiologistes (SCA), doivent s'engager à remédier à la pénurie de prestataires de soins de santé en anesthésie dans les régions rurales et éloignées. Ces organismes sont responsables de l'offre ou de la certification des programmes de formation et de formation continue. Ils participent également à l'établissement de lignes directrices pour les soins, qui aident à garantir la sécurité des services d'anesthésie. Le maintien des compétences cliniques peut indubitablement représenter un défi, surtout dans les régions éloignées. D'autres défis touchent à la bonne compréhension des besoins des prestataires d'anesthésie en région rurale pour assurer la pertinence du matériel éducatif fourni. L'investissement dans les nouvelles technologies, comme les plateformes en ligne, aiderait à résoudre certains de ces problèmes. La coordination des diverses organisations d'intervenants afin d'éviter le dédoublement du soutien ou, pire, une absence de soutien en raison d'une mauvaise communication entre les prestataires, est cruciale. Il sera essentiel de créer des occasions régulières de discussion entre les représentants des organismes provinciaux et nationaux et les fournisseurs de soins de santé locaux.

Une équipe sera mise sur pied pour créer un plan de travail basé sur les recommandations du symposium, pour ensuite le mettre en œuvre. Le Groupe consultatif de collaboration en anesthésiologie pour la pratique de la médecine générale et familiale (GCCA ou CAGA en anglais) est un organisme qui pourrait « héberger » un tel programme. Créé il y a plus de 20 ans pour répondre au besoin d'offrir davantage de services d'anesthésie dans les régions rurales du Canada, le GCCA a pour objectif de soutenir les médecins de famille en leur fournissant des compétences supplémentaires en anesthésie. Il s'agit d'un groupe multidisciplinaire composé de représentants de la SCA, du CMFC, de la Société de la médecine rurale du Canada (SMRC), de l'Association canadienne universitaire des départements d'anesthésie (ACUDA) et des directeurs des programmes de résidence en anesthésie en médecine familiale (omni-anesthésie). Conjointement financé par la SCA, le CMFC et la SMRC, le GCCA reconnaît clairement la nécessité d'un engagement et d'un investissement renouvelés dans les services ruraux.

Les efforts se sont poursuivis depuis le symposium. Par exemple, les anesthésiologistes de l'Hôpital Saint-Paul de Vancouver, en Colombie-Britannique, ont formé un partenariat avec des omni-anesthésistes du nord de la Colombie-Britannique pour offrir une formation par vidéoconférence à distance et en temps réel sur la réalisation de blocs nerveux du canal des adducteurs échoguidés, laquelle a été associée à une supervision en temps réel. ${ }^{8}$ Ce programme s'appuie sur un modèle robuste qui soutient la formation continue des omni-anesthésistes en Colombie-Britannique. ${ }^{9}$ Autre exemple : les Dres Ivy Bourgeault et Sarah Simkin et le Réseau canadien des personnels de santé ont entrepris une évaluation quantitative de la main-d'œuvre en anesthésie pour la période allant de 1996 à 2018, à l'aide des données de l'Institut canadien d'information sur la santé. ${ }^{10,11}$ En outre, les chercheurs du Sunnybrook Health Sciences Centre en Ontario ont créé une plateforme pour permettre le coaching en direct d'anesthésiologistes en région rurale et éloignée à l'aide d'un logiciel de télésimulation en réalité virtuelle et réalité augmentée appelé HoloSIM. ${ }^{12}$ Ces technologies permettent aux utilisateurs à distance d'apprendre d'enseignants situés dans un endroit géographique différent. Les salles d'opération peuvent être instantanément converties en centre de simulation hautefidélité en projetant des images holographiques interactives de patients. Les mises en situation peuvent être contrôlées et monitorées à distance par le formateur, réduisant ainsi les obstacles de matériel, de personnel et d'espace à la simulation. 
Dans l'ensemble, le mandat est clair. Néanmoins, des changements substantiels à la prestation d'anesthésie et de médecine de la douleur dans les régions rurales et éloignées du Canada ne seront pas possibles sans un financement provincial adéquat. De tels investissements doivent inclure l'augmentation du nombre de postes de formation pour les omni-anesthésistes et les anesthésiologistes spécialisés. En outre, les divers intervenants, notamment les gouvernements, ne doivent pas élaborer unilatéralement des solutions à la pénurie de prestataires de soins d'anesthésie sans consultation appropriée et significative des patients, des prestataires de soins de santé locaux et des groupes de médecins. Enfin, les départements universitaires doivent former des médecins qui s'engagent à travailler dans les communautés rurales. Des défis considérables nous attendent. Néanmoins, comme l'a fait remarquer la très honorable Julie Payette, alors gouverneure générale du Canada, dans son allocution d'ouverture du symposium : « Nous pouvons faire mieux, et nous sommes en fait mieux équipés que jamais pour résoudre ce problème, alors nous devons saisir toutes les occasions pour résoudre ce problème persistant. ${ }^{6}$

\section{Planification de la main-d'œuvre en anesthésie dans le Canada urbain et rural :}

Ivy Bourgeault, Mateen Raazi, Sarah Simkin, Pip Swartz

\section{Réseaux d'encadrement, mentorat et opportunités éducatives :}

Kirk McCarroll, Gary Morris, Elaine Ng, Margaret Tromp, Hira Raheel

\section{Soutien des politiques destinées à l'anesthésie et la} médecine de la douleur en région rurale :

Lee Errett, Dietrich Furstenburg, Vaibhav Kamble, John McAlpine, Taryn Davidson

\section{Technologie, réalité virtuelle, encadrement et} apprentissage à distance :

Fahad Alam, Sarah Newbery, Ahtsham Niazi, Julian Wiegelmann, Julian DeBacker
Formation et soutien aux médecins généralistes ruraux (anesthésie, obstétrique, chirurgie) :

Ryan Falk, Stu Iglesias, Aviva Stewart, Enrich Van der Linde, Miguel Proulx

Modèles de soins en milieu rural et éloigné au Canada, incluant les professionnels paramédicaux :

Roy Kirkpatrick, Matthew Kurrek, Jason McVicar, Roanne Preston, Alborz Noorani

Élaboration des politiques par collaboration avec les sociétés médicales nationales et le gouvernement :

Angela Enright, Colin McCartney, Robert Milkovich, Victor Ng, Tarimobo Otobo

Disclosures None.

Funding statement Existing resources from the Departments of Anesthesiology and Pain Medicine, Temerty Faculty of Medicine, University of Toronto, Toronto, Canada provided funding for this work.

Editorial responsibility This submission was handled by Dr. Stephan K.W. Schwarz, Editor-in-Chief, Canadian Journal of Anesthesia.

Déclaration Aucune.

Déclaration de financement Les ressources existantes des départements d'anesthésiologie et de médecine de la douleur de la Faculté de médecine Temerty de l'Université de Toronto, Toronto, Canada, ont financé ces travaux.

Responsabilité éditoriale Ce manuscrit a été traité par Dr Stephan K.W. Schwarz, rédacteur en chef, Journal canadien d'anesthésie.

\section{References}

1. McVicar JA, Poon A, Caron NR, et al. Postoperative outcomes for Indigenous Peoples in Canada: a systematic review. CMAJ 2021; 193: E713-22.

2. Smylie J, O'Brien K, Beaudoin E, et al. Long-distance travel for birthing among Indigenous and non-Indigenous pregnant people in Canada. CMAJ 2021; 193: E948-55.

3. Society of Rural Physicians of Canada; College of Family Physicians of Canada.The Rural Road Map. Available from URL: https://srpc.ca/Rural_Road_Map_Directions (accessed July 2021).

4. Orser BA, Wilson CR. Canada needs a national strategy for anesthesia services in rural and remote regions. CMAJ 2020; 192: E861-3. 
5. CBC News. Wait-list for children's dental surgery in Nunavut has doubled to 1,000. Available from URL: https://www.cbc.ca/ news/canada/north/nunavut-dental-surgery-1.6090357 (accessed July 2021).

6. Anesthesiology \& Pain Medicine, University of Toronto. Anesthesia Care \& Pain in Rural and Remote Regions of Canada Symposium. Available from URL: https://www. anesthesia.utoronto.ca/anesthesia-care-pain-medicine (accessed July 2021).

7. Orser BA, Wilson RC, Co-Chairs. Symposium on Anesthesia Care and Pain Medicine in Rural and Remote Regions of Canada; Nov 20-21, 2020. Available from URL: https://www.anesthesia. utoronto.ca/sites/default/files/anesthesia_symposium_report_2020_ final_report_posted_on_website.pdf (accessed July 2021).

8. Rural Coordination Centre of BC. BC Rural Update Newsletter. Available from URL: https://rccbc.ca/rccbc/about-rccbc/ (accessed July 2021).
9. Rural Coordination Centre of BC. BC Rural Update. Surgical Obstetrical Networks (RSON). Available from URL: https:// enews.rccbc.ca/2021/01/29/fpa-ecoaching/ (accessed July 2021).

10. Canadian Health Workforce Network. Available from URL: https://www.hhr-rhs.ca/en/ (accessed July 2021).

11. Bourgeault I, Simkin S, Chamberland-Rowe $C$. Poor health workforce planning is costly, risky and inequitable. CMAJ 2019; 191: E1147-8.

12. Wiegelmann J, Alam F. HoloSIM. Available from URL: https:// holosim.ca/ (accessed July 2021).

Publisher's Note Springer Nature remains neutral with regard to jurisdictional claims in published maps and institutional affiliations. 